\title{
Linear Feedback Synchronization Used in the Three-Dimensional Duffing System
}

\author{
Jian-qun Han, ${ }^{1}$ Xu-dong Shi, ${ }^{2}$ and Hong Sun ${ }^{3}$ \\ ${ }^{1}$ College of Engineering, Bohai University, Jinzhou 121013, China \\ ${ }^{2}$ Tianjin Key Laboratory for Civil Aircraft Airworthiness and Maintenance Civil Aviation University of China, Tianjin 300300, China \\ ${ }^{3}$ Higher Vocational College, Bohai University, Jinzhou 121013, China \\ Correspondence should be addressed to Jian-qun Han; hanjianqun@126.com
}

Received 17 January 2015; Accepted 2 June 2015

Academic Editor: Petko Petkov

Copyright (C) 2015 Jian-qun Han et al. This is an open access article distributed under the Creative Commons Attribution License, which permits unrestricted use, distribution, and reproduction in any medium, provided the original work is properly cited.

\begin{abstract}
It has been realized that synchronization using linear feedback control method is efficient compared to nonlinear feedback control method due to the less computational complexity and the synchronization error. For the problem of feedback synchronization of Duffing chaotic system, in the paper, we firstly established three-dimensional Duffing system by method of variable decomposition and, then, studied the synchronization of Duffing chaotic system and designed the control law based on linear feedback control and Lyapunov stability theory. It is proved theoretically that the two identical integer order chaotic systems are synchronized analytically and numerically.
\end{abstract}

\section{Introduction}

In recent years, the chaotic control has become one of the important research fields of nonlinear science and received the attention of many scholars around the world. The possibility of synchronizing two chaotic systems has been introduced by Pecora and Carroll [1] and the synchronization of two identical chaotic systems with different initial conditions has been presented in [2]. Moreover, synchronization of two chaotic systems has been studied extensively in the last few years. Most recently, the problem of controlling chaos for new dynamical system has been studied and the sufficient conditions for synchronization of chaotic systems have been derived in [3]. An efficient nonlinear control method has been applied to the synchronization of unified chaotic systems using the Lyapunov method in [4] and a nonlinear control scheme for the synchronization has been presented using the Lyapunov stability theory in [5]. The synchronization of an energy resource system has been investigated and three linear control schemes have been proposed to synchronize an energy resource system in [6]. The synchronization process of a four-dimensional chaotic system by using linear feedback controller, single variable, and adaptive controller methods has been proposed and demonstrated in [7]. Synchronization of energy resource systems has been proposed when the parameters of the master system are unknown and different from the slave system using adaptive linear feedback control in $[8]$.

Chaos synchronization has been of tremendous worldwide interest in communication systems, which has applications in the encryption and decryption of information for secure communications. An adaptive scheme has been exhibited in [9] for chaos synchronization that solves the problem of security in the communications. The authors in [10] have designed secure digital communication systems using chaotic modulation, cryptography, and chaotic synchronization and their security features have been analyzed. Two methods of encoding and decoding message for secure communication based on an adaptive chaos synchronization have been investigated by Xing and Huang [11]. In [12], a new technique has been suggested for synchronizing two chaotic systems and that technique has been applied to digital cryptography [13] for sending and receiving messages.

At present, the main research methods of chaotic control are OGY and feedback control method [14, 15]. The chaos can be controlled by constructing simple fractional 
order controller in [16]. The chaos synchronization between two different chaotic systems can be realized based on the nonlinear feedback control method in [17]. Feedback technology is commonly used in engineering, and the use of this technology can realize the control of chaotic systems, such as changing unstable fixed point into stable one and controlling the periodic orbit. Chaos synchronization can be regarded as a kind of control that the controlled chaotic system moves in the target system orbit. Generally speaking, feedback methods used in the chaos synchronization can be divided into parameters feedback method and state variable feedback method. Output feedback and linear feedback synchronization of chaotic systems are, respectively, studied in $[18-21]$.

Study of chaotic oscillator characteristic is an important subject. Many scientific researchers are interested in it [2225]. However, less research is focused on the problem of Duffing chaotic system control. Therefore, based on the threedimensional Duffing system and the theory of Lyapunov equation of system stability judgment, the linear state feedback synchronization in Duffing chaotic system is studied in this paper.

\section{Three-Dimensional Duffing System}

There are many problems in engineering such as packaging systems based on displacement excitation of nonlinear vibrations and pressure sensors nonlinear vibration and so they can be simplified into a forced Duffing equation with cubic nonlinearity, governed by

$$
\ddot{x}(t)+\mu \dot{x}(t)+\omega_{0}^{2} x(t)+\varepsilon x(t)^{3}=F \cos \Omega t,
$$

where $x(t)$ is the solution of (1), $\mu, \omega_{0}, \varepsilon, F, \Omega$ are real constants, and the dot represents differentiation with respect to $t$.

Definition 1. The particular form of Duffing system related to system (1) is described as

$$
\begin{aligned}
& \dot{x}=y, \\
& \dot{y}=x-x^{3}-k y+r \cos \omega t,
\end{aligned}
$$

where $r, \omega$ are the amplitude and frequency of driving force, $k$ is damping ratio, and $x-x^{3}$ is the nonlinear restoring force.

When given the system initial states $x(0)=0$ and $y(0)=$ 1 in (2), two-dimensional Duffing system under sinusoidal signal drive produces the chaotic phase diagram shown in Figure 1.

Definition 2. Given a constant $b$, system (2) can be converted into the following state equation:

$$
\begin{aligned}
& \dot{x}=y, \\
& \dot{y}=-k y-z+b+r \cos \omega t, \\
& \dot{z}=3 x^{2} y-y .
\end{aligned}
$$

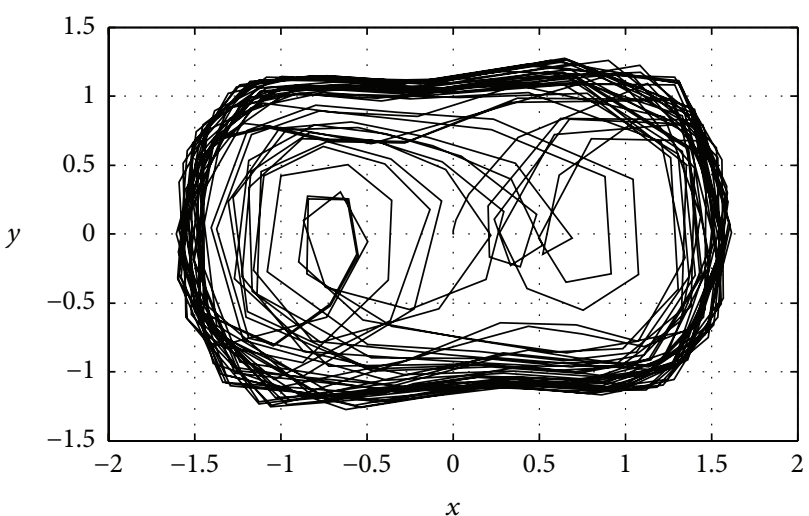

FIGURE 1: 2D Duffing chaotic system phase diagram.

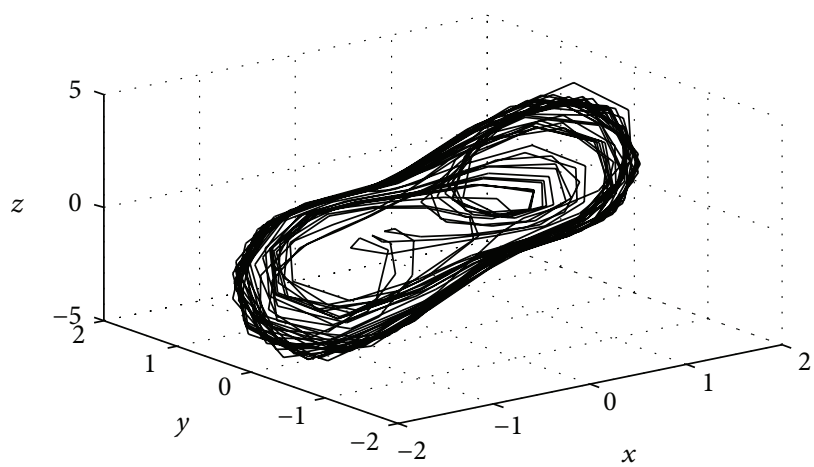

Figure 2: 3D Duffing chaotic system phase diagram.

Proof. Choose the following differential equation:

$$
\dot{z}=3 x^{2} y-y .
$$

Integrating $\dot{z}$ taken in (4) into system (2) yields

$$
z=x^{3}-x+c,
$$

where $c$ is undetermined constant and the value can be determined by the system initial state.

Remark 3. From the above analysis, it follows that $c$ and $b$ are unbounded, but $b$ is used to counteract the effects of $c$. When given $x(0)=0$ and $z(0)=0$ in system (3), $c=0$ and $b=0$ hold. The three-dimensional Duffing chaotic dynamic system (3) is the same as system (2). Under the general conditions, system (2) can be modified to system (3) by adjusting variable $b$ to offset the impact of variable $c$.

When given $x(0)=0, y(0)=1, z(0)=0$, and $b=0$ in (3), three-dimensional Duffing system produces the chaotic phase diagram shown in Figure 2. Its plane projections on the $x o y, y o z$, and $x o z$ are shown in Figures 3, 4, and 5. By comparing Figures 1 and 3, we can obtain the result that twodimensional Duffing system and three-dimensional Duffing system are consistent. Figures 4 and 5 can only produce in three-dimensional Duffing system.

The simulation results of equivalent condition are the premise of system initial states $x(0)=0$ and $z(0)=0$; 


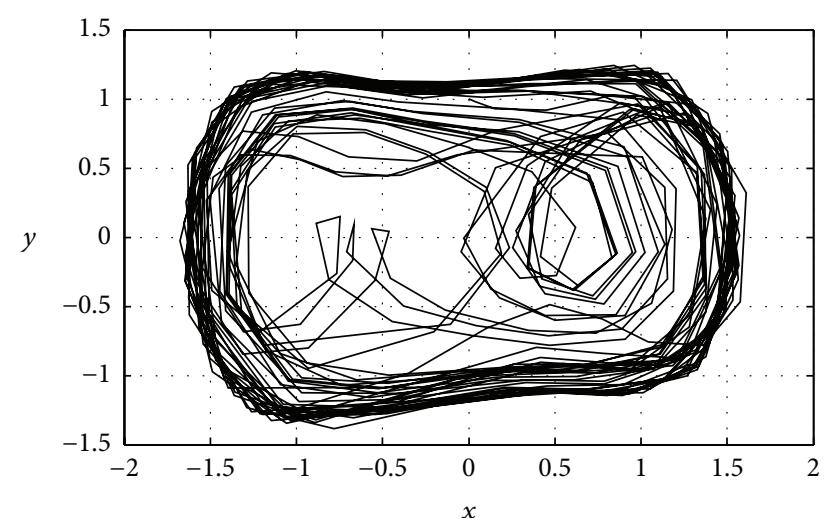

FIGURE 3: 3D Duffing chaotic system phase diagram in the xoy plane projection.

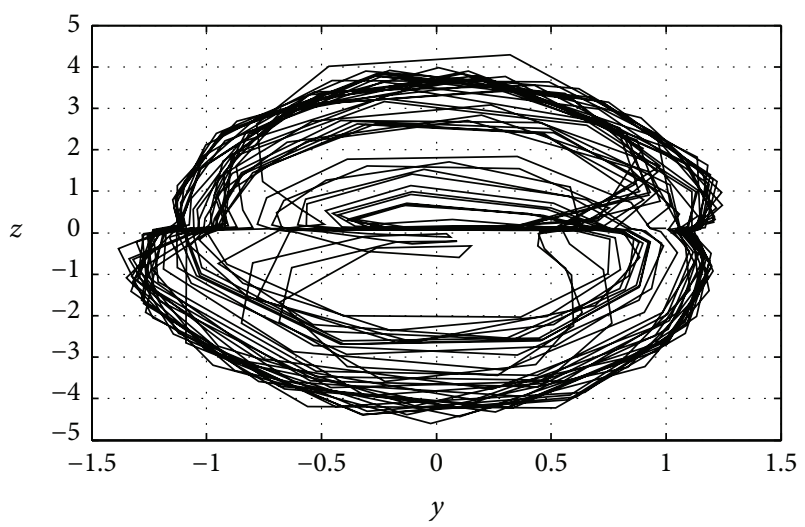

FIGURE 4: 3D Duffing chaotic system phase diagram in the yoz plane projection.

otherwise the system status may not be chaotic. When given $x(0)=0, y(0)=1$, and $z(0)=2$, simulation result is shown in Figure 6. The system state is not chaotic.

When assuming $b=2$ in (3), the system phase diagram as shown in Figure 7 is chaotic. From the above analysis, results counteracting the effects of initial condition on system state and keeping the system chaotic can be obtained by adjusting the variable $b$.

\section{State Feedback Synchronization in Duffing System}

Definition 4. Given three positive constants $k_{1}, k_{2}$, and $k_{3}$, the response system of state feedback synchronization in Duffing system can be written as

$$
\begin{aligned}
& \dot{\hat{x}}=\widehat{y}-k_{1}(\widehat{x}-x), \\
& \dot{\hat{y}}=\widehat{x}-k \hat{y}-\widehat{z}-k_{2}(\hat{y}-y)+r \cos \omega t, \\
& \dot{\vec{z}}=3 \widehat{x}^{2} \hat{y}-k_{3}(\widehat{z}-z) .
\end{aligned}
$$

When driven by the state error feedback, the response system can keep pace with the drive system.

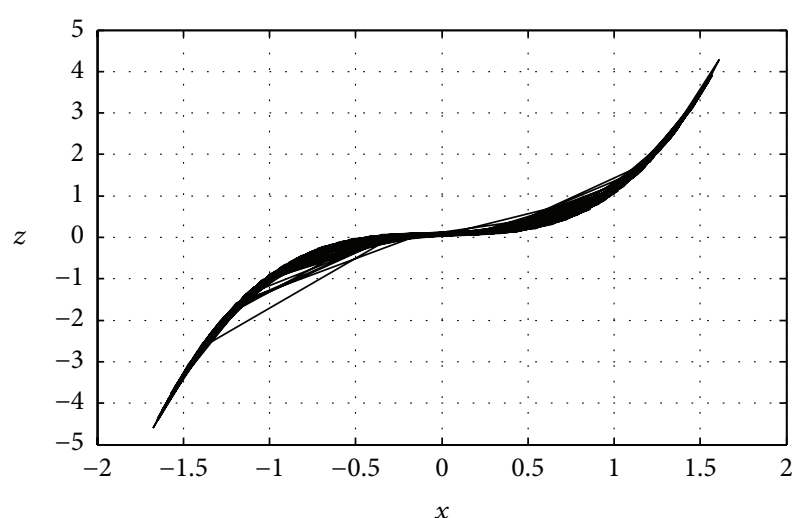

FIGURE 5: 3D Duffing chaotic system phase diagram in the $x o z$ plane projection.

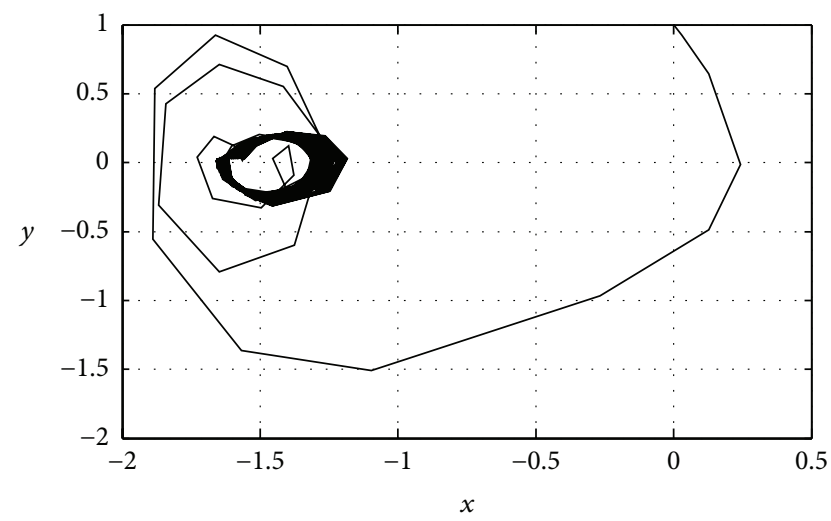

FIGURE 6:3D Duffing chaotic system phase diagram in the xoy plane projection.

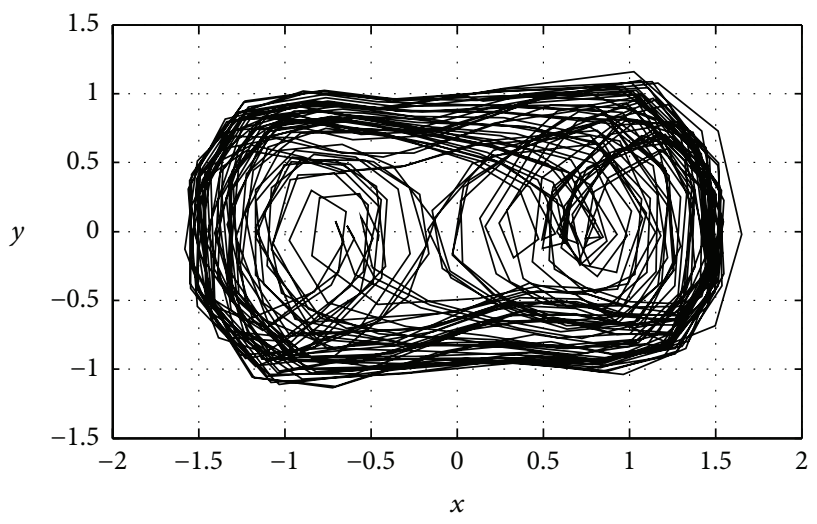

FIGURE 7:3D Duffing chaotic system phase diagram in the xoy plane projection.

Proof. Constructed from drive and response system, the error system can be described as

$$
\begin{aligned}
& \dot{x}-\dot{\hat{x}}=y-\widehat{y}+k_{1}(\widehat{x}-x), \\
& \dot{y}-\dot{\hat{y}}=x-\widehat{x}-k(y-\widehat{y})-(z-\widehat{z})+k_{2}(\hat{y}-y), \\
& \dot{z}-\dot{\vec{z}}=3 x^{2} y-3 \hat{x}^{2} \hat{y}+k_{3}(\widehat{z}-z) .
\end{aligned}
$$




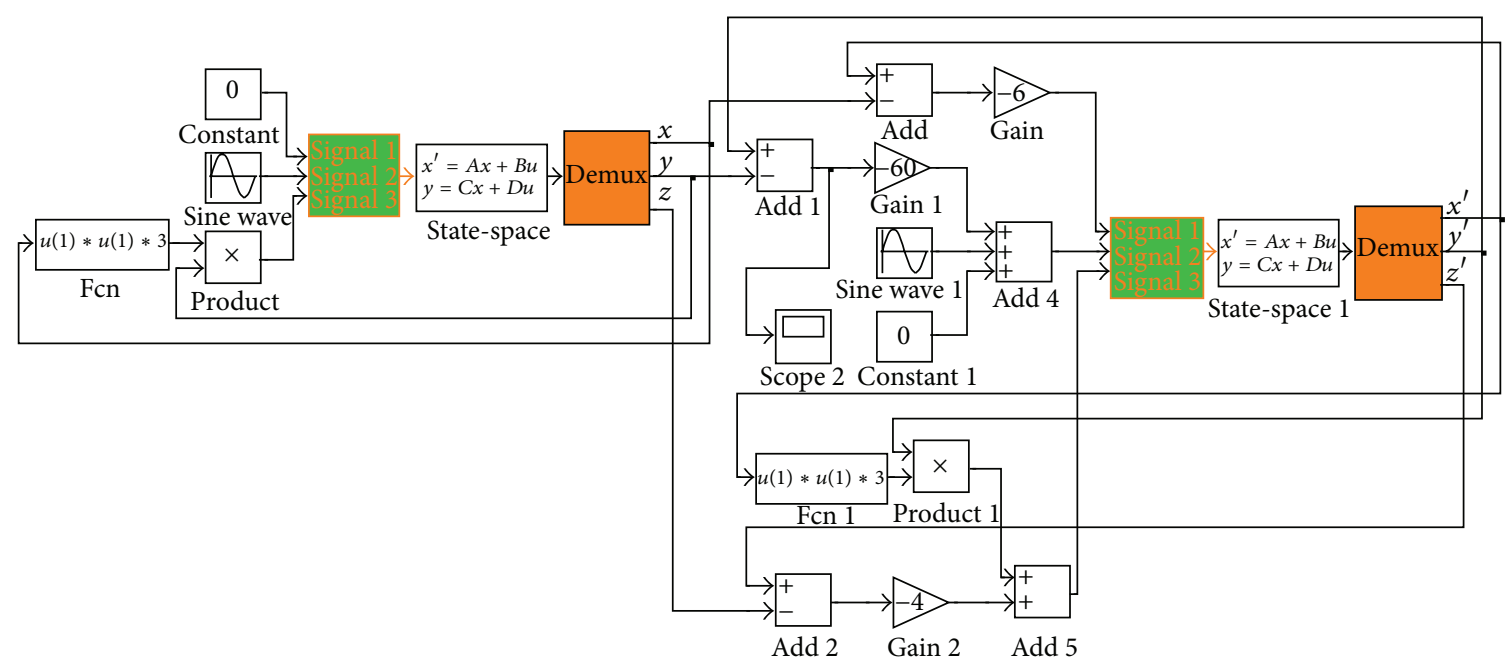

FIGURE 8: State feedback simulation system.

Let $e_{x}=x-\widehat{x}, e_{y}=y-\hat{y}$, and $e_{z}=z-\widehat{z}$. Then (7) can change into

$$
\begin{aligned}
& \dot{e}_{x}=e_{y}-k_{1} e_{x}, \\
& \dot{e}_{y}=e_{x}-\left(k+k_{2}\right) e_{y}-e_{z}, \\
& \dot{e}_{z}=3 x^{2} y-3 \hat{x}^{2} \hat{y}-k_{3} e_{z} .
\end{aligned}
$$

Now choose (9) as Lyapunov function:

$$
V=\frac{1}{2}\left(e_{x}^{2}+e_{y}^{2}+e_{z}^{2}\right)
$$

From (8) and differentiating (9), we can obtain

$$
\begin{aligned}
\dot{V}= & e_{x} \dot{e}_{x}+e_{y} \dot{e}_{y}+e_{z} \dot{e}_{z} \\
= & 2 e_{x} e_{y}-k_{1} e_{x}^{2}-\left(k+k_{2}\right) e_{y}^{2}-e_{y} e_{z}-k_{3} e_{z}^{2} \\
& +\left(3 x^{2} y-3 \hat{x}^{2} \hat{y}\right) e_{z} .
\end{aligned}
$$

Considering that Duffing chaotic system is bounded, $3 x^{2}$ and $3 \widehat{x}^{2}$ are not bigger than negative $M^{2}$. Then (10) can be modified as

$$
\begin{aligned}
\dot{V}= & 2 e_{x} e_{y}-k_{1} e_{x}^{2}-\left(k+k_{2}\right) e_{y}^{2}+\left(M^{2}-1\right) e_{y} e_{z} \\
& -k_{3} e_{z}^{2} .
\end{aligned}
$$

If $\dot{V}<0$ in (11), (8) at the origin is asymptotically stable. The coefficients of $k_{1}, k_{2}$, and $k_{3}$ in (11) should satisfy the following conditions:

$$
\begin{aligned}
& \text { (1) } \sqrt{k_{1}} \cdot \sqrt{\left(k+k_{2}\right) / 2}>1, \forall k_{1}>0 \text { and } k_{2}>0 . \\
& \text { (2) } \sqrt{k_{3}} \cdot \sqrt{\left(k+k_{2}\right) / 2}>\left(M^{2}-1\right) / 2, \forall k_{3}>0 .
\end{aligned}
$$

Remark 5. The state feedback system based on (6) is shown in Figure 8. The initial states of drive system are $x(0)=0.5$,

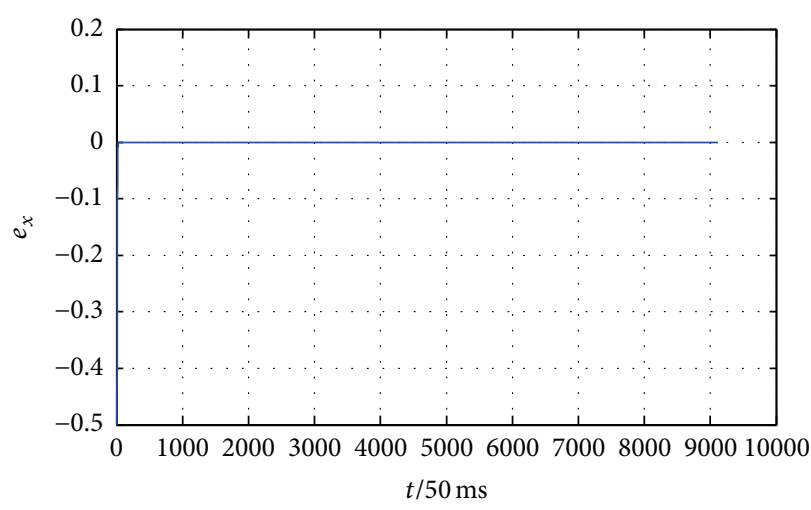

FIGURE 9: The curve $e_{x}$ for multivariable feedback system.

$y(0)=1$, and $z(0)=2$ in (5). The initial states of response system are $\widehat{x}(0)=0, \widehat{y}(0)=1$, and $\widehat{z}(0)=0$ in (6). According to phase diagram of chaos Duffing system, we can know $M^{2} \leq 4$. Utilizing the above conditions in (7) with coefficients $k_{1}=6, k_{2}=60$, and $k_{3}=4$, feedback coefficients $k_{1}, k_{2}$, and $k_{3}$ are to meet the coefficient value range in Section 3 . Let $e_{x}=x-\widehat{x}, e_{y}=y-\widehat{y}$, and $e_{z}=z-\widehat{z}$. From (11), we can obtain $\dot{V}<0$. According to (8), the simulation results based on state feedback method are shown in Figures 9, 10, and 11. Error curves tend to zero. Simulation results show that drive and response system can keep the good synchronization performance.

\section{Reduction of State Feedback Terms}

Multivariable feedback can realize synchronization of two chaotic systems. But, for many chaotic systems, only single variable signal feedback can achieve synchronization between systems. Feedback coefficient in Section 3 can be further deduced as follows. 


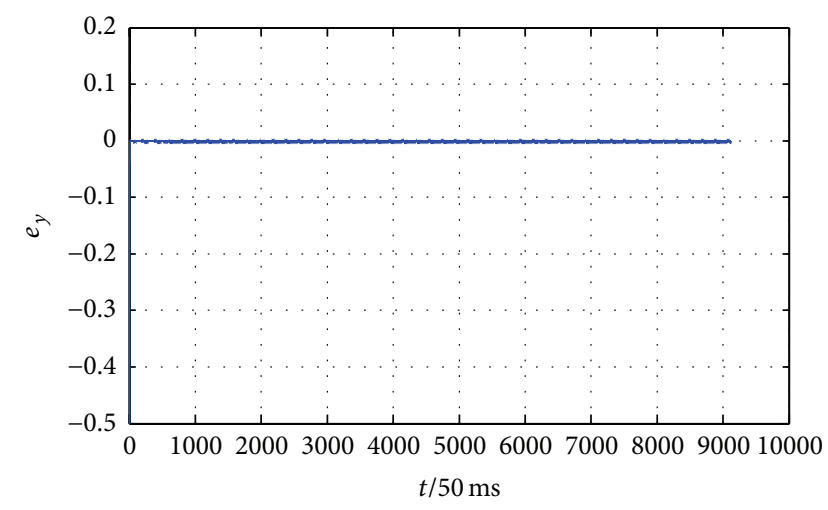

FIgURE 10: The curve $e_{y}$ for multivariable feedback system.

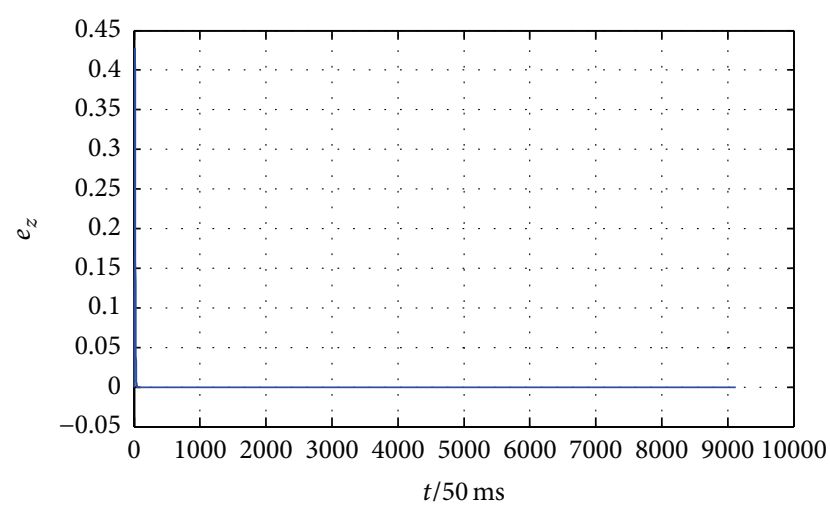

Figure 11: The curve $e_{z}$ for multivariable feedback system.

Proof. Let $k_{2}=0$ and $k_{3}=0$ in (6). The response system of state feedback synchronization in Duffing system can be modified to (12), where $k_{1}$ is a feedback coefficient. Consider

$$
\begin{aligned}
& \dot{\hat{x}}=\widehat{y}-k_{1}(\widehat{x}-x), \\
& \dot{\hat{y}}=\widehat{x}-k \hat{y}-\widehat{z}+r \cos \omega t, \\
& \dot{\hat{z}}=3 \widehat{x}^{2} \widehat{y} .
\end{aligned}
$$

According to (12) and stability proof for the error system in Section 3, we can obtain (13) from (11):

$$
\dot{V}=2 e_{x} e_{y}-k_{1} e_{x}^{2}-k e_{y}^{2}+\left(M^{2}-1\right) e_{y} e_{z}
$$

From (4), we know the symbol of $e_{z}$ is the same as that of $e_{x}$; namely, $e_{z} \propto e_{x}$. Equation (13) can be modified as

$$
\dot{V}=2 e_{x} e_{y}-k_{11} e_{x}^{2}-k e_{y}^{2}+\left(M^{2}-1\right) e_{y} e_{z}-k_{12} e_{z}^{2}
$$

where $k_{11}$ and $k_{12}$ satisfy

$$
k_{11} e_{x}^{2}+k_{12} e_{z}^{2}=k_{1} e_{x}^{2}
$$

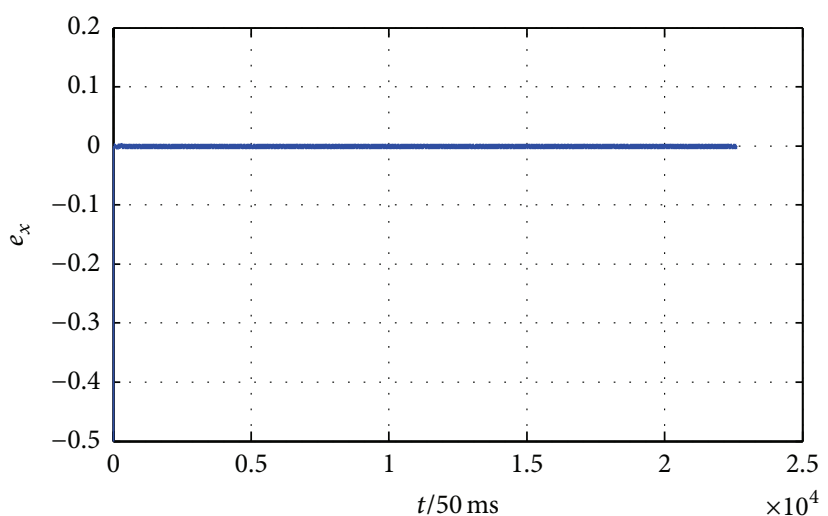

FIGURE 12: The curve $e_{x}$ for single variable feedback system.

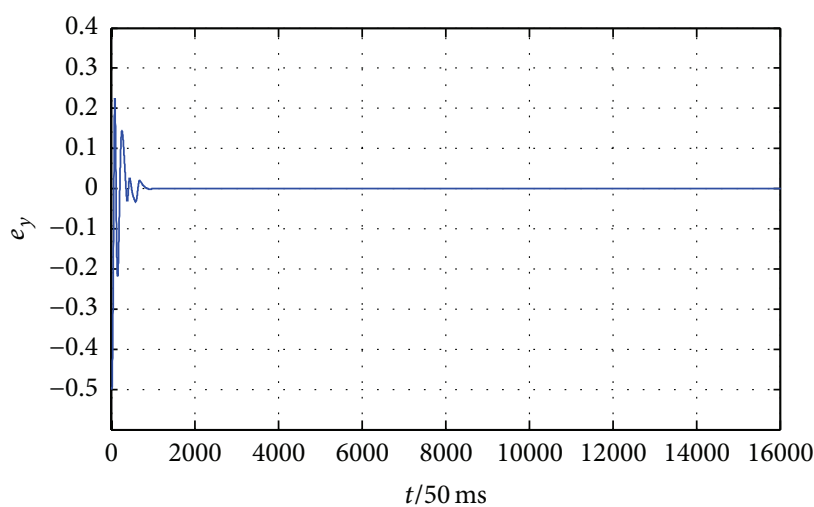

FIGURE 13: The curve $e_{y}$ for single variable feedback system.

If $\dot{V}<0$ in (14), $k_{11}$ and $k_{12}$ should satisfy the following conditions:

(3) $\sqrt{k_{11}} \cdot \sqrt{k / 2}>1, \forall k_{11}>0$.

(4) $\sqrt{k_{12}} \cdot \sqrt{k / 2}>\left(M^{2}-1\right) / 2, \forall k_{12}>0$.

Remark 6. From (15), we know that when feedback coefficient $k_{1}$ is big enough, conditions (3) and (4) are satisfied. Let $k_{1}=100$ and select the initial states in (12) similar to (6); then, like that in Section 3, the error of drive and response system tends to zero, and the two systems can keep synchronization too. According to (12), the simulation results based on single variable feedback method are shown in Figures 12, 13, and 14. Error curves tend to zero.

\section{Conclusions}

Linear and nonlinear feedback controllers can be designed to realize drive-response synchronization of an existing chaotic system. It has shown that synchronization using linear feedback control method is suitable and efficient compared to nonlinear control method due to less synchronization cost and synchronization error. The present work has studied a new three-dimensional chaotic synchronization of Duffing 


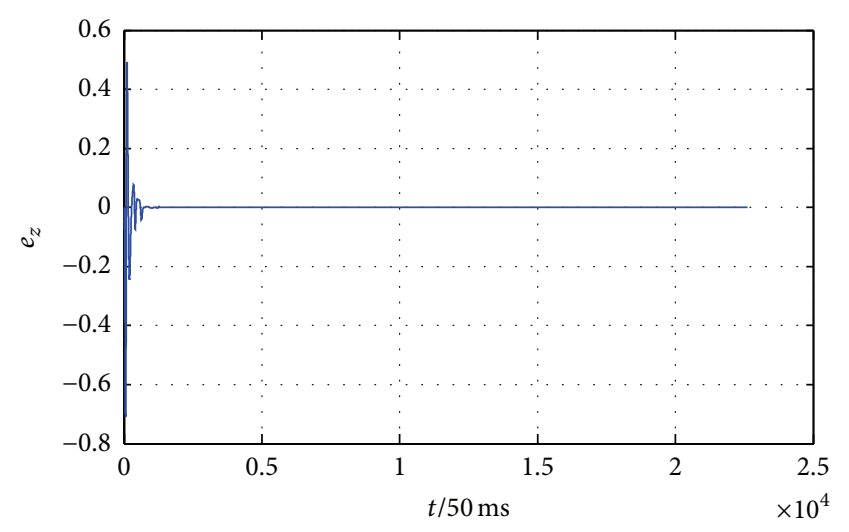

FIGURE 14: The curve $e_{z}$ for single variable feedback system.

system. On the basis of three-dimensional model, a linear state feedback method is used and has successfully driven the state of response system to zero. This method is simple and practical and can select control parameters in considerable range. In addition, three-dimensional Duffing system is equivalent to two-dimensional system but can show more information, so the structure of new Duffing system is worth future studying of weak signal detection and secret communication.

\section{Conflict of Interests}

The authors declare that there is no conflict of interests regarding the publication of this paper.

\section{Acknowledgment}

This work is supported by the National Natural Science Foundation of China under Grant Contract 51277011.

\section{References}

[1] L. M. Pecora and T. L. Carroll, "Synchronization in chaotic systems," Physical Review Letters, vol. 64, no. 8, pp. 821-824, 1990.

[2] T. L. Carroll and L. M. Pecora, "Synchronizing chaotic circuits," IEEE Transactions on Circuits and Systems, vol. 38, no. 4, pp. 453-456, 1991.

[3] M. T. Yassen, "Controlling chaos and synchronization for new chaotic system using linear feedback control," Chaos, Solitons \& Fractals, vol. 26, no. 3, pp. 913-920, 2005.

[4] J. H. Park, "On synchronization of unified chaotic systems via nonlinear control," Chaos, Solitons and Fractals, vol. 25, no. 3, pp. 699-704, 2005.

[5] J. H. Park, "Adaptive controller design for modified projective synchronization of Genesio-Tesi chaotic system with uncertain parameters," Chaos, Solitons and Fractals, vol. 34, no. 4, pp. 11541159, 2007.

[6] Z. Wang, "Chaos synchronization of an energy resource system based on linear control," Nonlinear Analysis. Real World Applications, vol. 11, no. 5, pp. 3336-3343, 2010.
[7] X. Wang and Y. Wang, "Adaptive control for synchronization of a four-dimensional chaotic system via a single variable," Nonlinear Dynamics, vol. 65, no. 3, pp. 311-316, 2011.

[8] X. Shi and Z. Wang, "Adaptive synchronization of the energy resource systems with mismatched parameters via linear feedback control," Nonlinear Dynamics, vol. 69, no. 3, pp. 993-997, 2012.

[9] S. Boccaletti, A. Farini, and F. T. Arecchi, "Adaptive synchronization of chaos for secure communication," Physical Review E, vol. 55, no. 5, pp. 4979-4981, 1997.

[10] T.-I. Chien and T.-L. Liao, "Design of secure digital communication systems using chaotic modulation, cryptography and chaotic synchronization," Chaos, Solitons and Fractals, vol. 24, no. 1, pp. 241-255, 2005.

[11] G.-J. Xing and D.-B. Huang, "Encoding-decoding message for secure communication based on adaptive chaos synchronization," Journal of Shanghai University (English Edition), vol. 12, no. 5, pp. 400-404, 2008.

[12] S. Banerjee and A. R. Chowdhury, "Lyapunov function, parameter estimation, synchronization and chaotic cryptography," Communications in Nonlinear Science and Numerical Simulation, vol. 14, no. 5, pp. 2248-2254, 2009.

[13] M. Mitra and S. Banerjee, "Digital cryptography and feedback synchronization of chaotic systems," International Journal of Modern Physics B, vol. 25, no. 4, pp. 521-529, 2011.

[14] M. U. Akhmet and M. O. Fen, "Chaotic period-doubling and OGY control for the forced Duffing equation," Communications in Nonlinear Science and Numerical Simulation, vol. 17, no. 4, pp. 1929-1946, 2012.

[15] G. X. Chen, "A simple adaptive feedback control method for chaos and hyper-chaos control," Applied Mathematics and Computation, vol. 217, no. 17, pp. 7258-7264, 2011.

[16] M. S. Tavazoei, M. Haeri, S. Bolouki, and M. Siami, "Using fractional-order integrator to control chaos in single-input chaotic systems," Nonlinear Dynamics, vol. 55, no. 1-2, pp. 179190, 2009.

[17] H.-H. Chen, G.-J. Sheu, Y.-L. Lin, and C.-S. Chen, "Chaos synchronization between two different chaotic systems via nonlinear feedback control," Nonlinear Analysis: Theory, Methods \& Applications, vol. 70, no. 12, pp. 4393-4401, 2009.

[18] H. Salarieh and M. Shahrokhi, "Multi-synchronization of chaos via linear output feedback strategy," Journal of Computational and Applied Mathematics, vol. 223, no. 2, pp. 842-852, 2009.

[19] H. K. Lam, "Output-feedback synchronization of chaotic systems based on sum-of-squares approach," Chaos, Solitons and Fractals, vol. 41, no. 5, pp. 2624-2629, 2009.

[20] F. Wang and C. Liu, "A new criterion for chaos and hyperchaos synchronization using linear feedback control," Physics Letters, Section A: General, Atomic and Solid State Physics, vol. 360, no. 2, pp. 274-278, 2006.

[21] Z. Y. Yan and P. Yu, "Linear feedback control, adaptive feedback control and their combination for chaos (lag) synchronization of LC chaotic systems," Chaos, Solitons \& Fractals, vol. 33, no. 2, pp. 419-435, 2007.

[22] W. L. Zhao, J. X. Zhao, Z. Q. Huang, and Q. H. Meng, "Weak signal detection technology based on Holmes Duffing oscillator," Procedia Engineering, vol. 29, pp. 1796-1802, 2012.

[23] G. Wang, W. Zheng, and S. He, "Estimation of amplitude and phase of a weak signal by using the property of sensitive dependence on initial conditions of a nonlinear oscillator," Signal Processing, vol. 82, no. 1, pp. 103-115, 2002. 
[24] N. Q. Hu and X. S. Wen, “The application of Duffing oscillator in characteristic signal detection of early fault," Journal of Sound and Vibration, vol. 268, no. 5, pp. 917-931, 2003.

[25] W. Zhang and B.-R. Xiang, "A Duffing oscillator algorithm to detect the weak chromatographic signal," Analytica Chimica Acta, vol. 585, no. 1, pp. 55-59, 2007. 

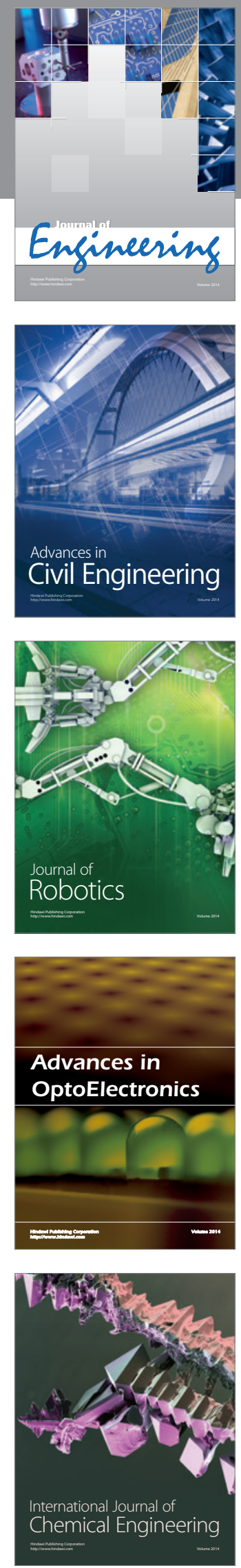

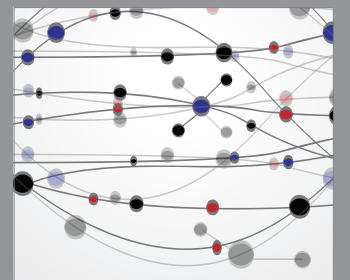

The Scientific World Journal
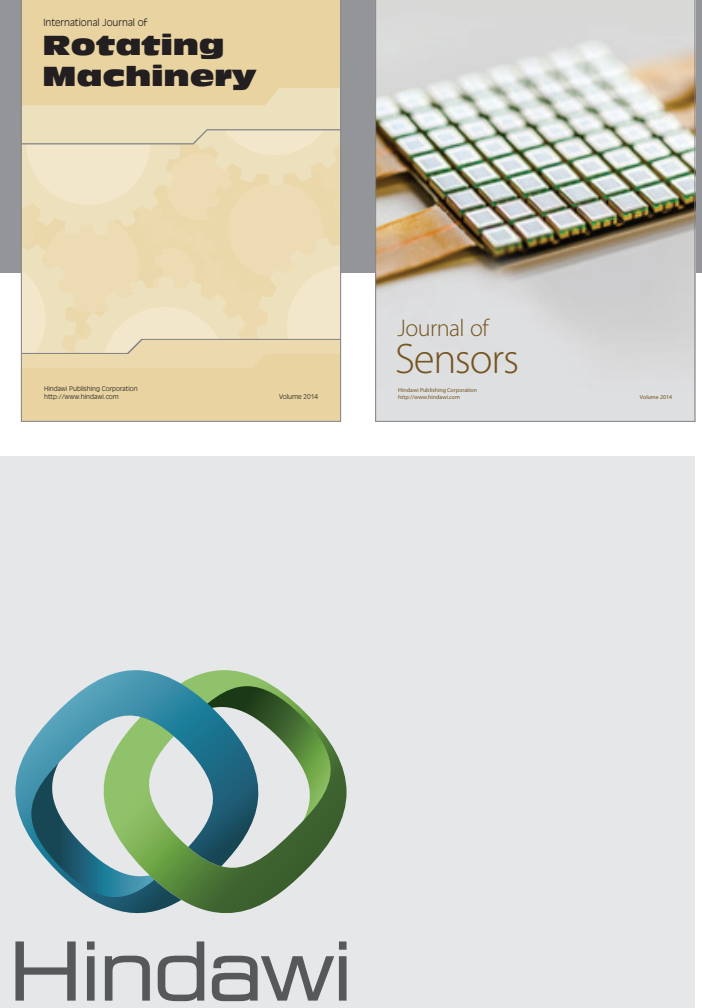

Submit your manuscripts at http://www.hindawi.com
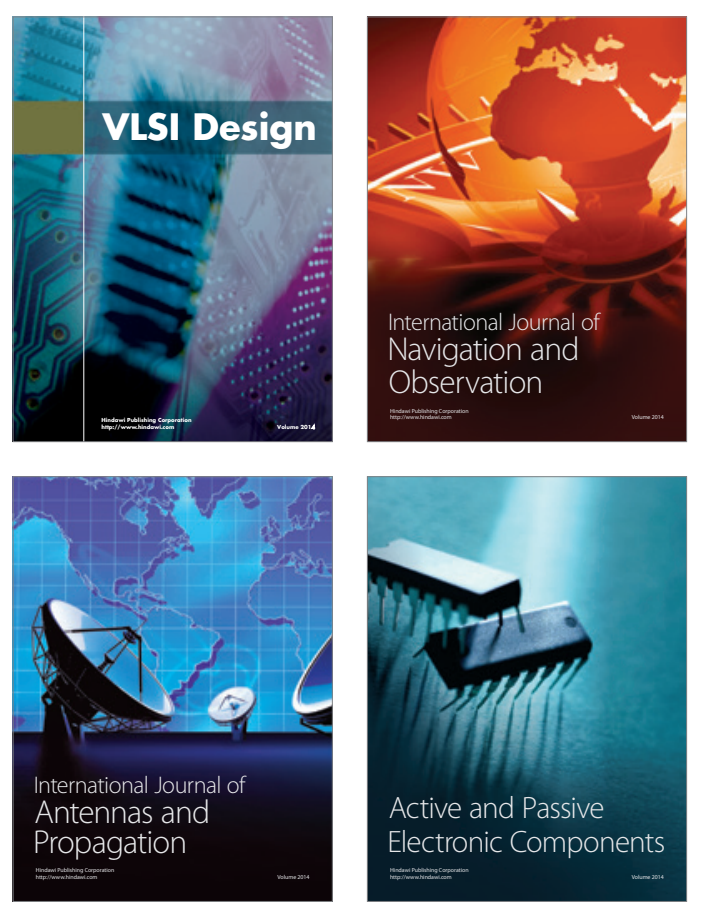
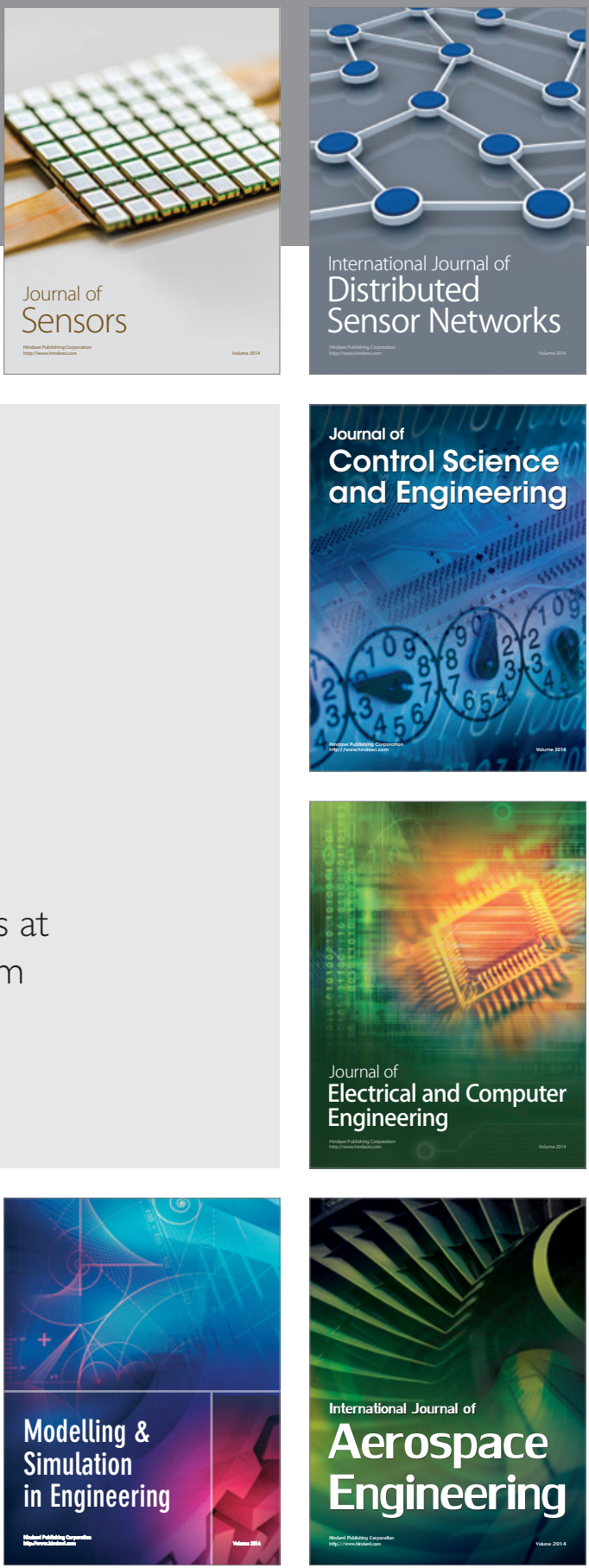

Journal of

Control Science

and Engineering
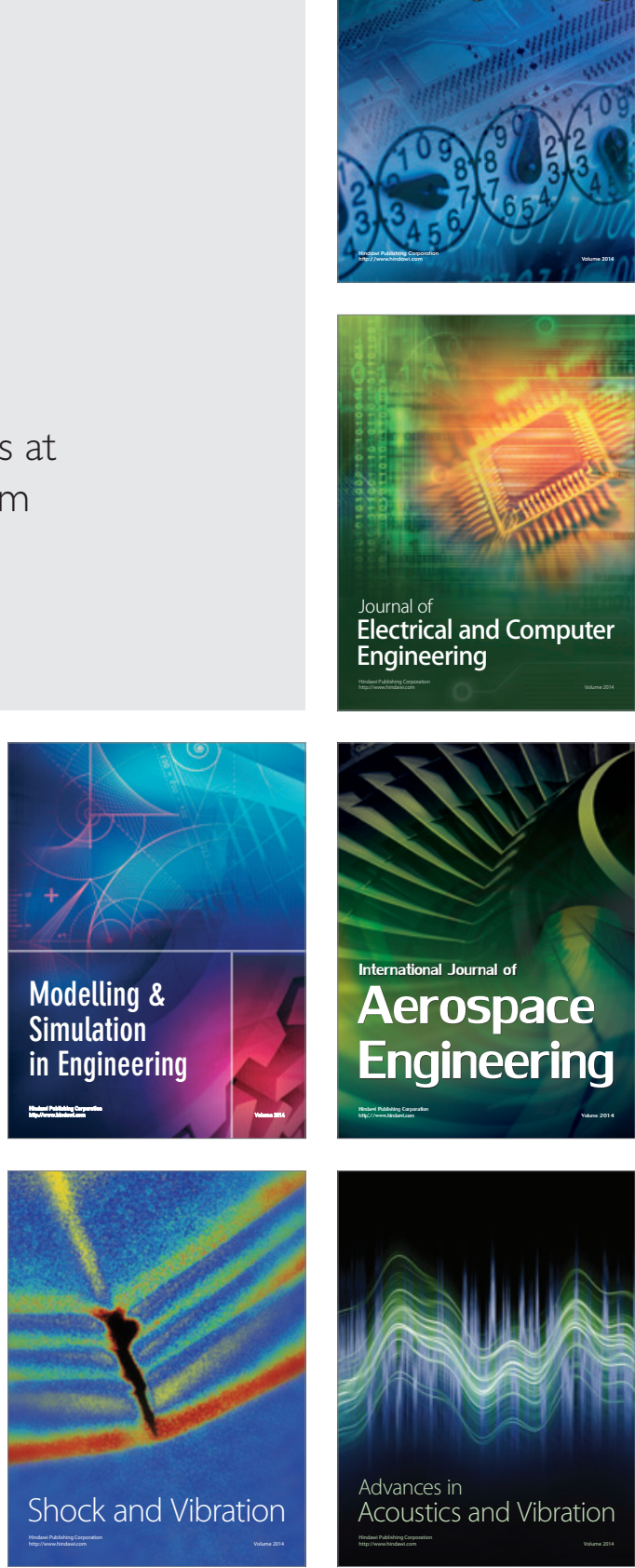